\title{
EFEITOS DA PRÁTICA DE EXERCÍCIO DE DUPLA TAREFA EM IDOSOS COM DOENÇA DE ALZHEIMER: REVISÃO SISTEMÁTICA
}

Jéssica Fernanda Siqueira

Graduada em Educação física (UNIFAMMA)), Brasil.

Mateus Dias Antunes

Doutorando em Ciências da Reabilitação na Universidade de São Paulo (USP), Brasil.

\section{José Roberto A. do Nascimento Júnior}

Doutor em Educação Física. Universidade Federal do

\section{Daniel Vicentini Oliveira}

Doutor em Gerontologia (UNICAMP). Centro Universitário Metropolitano de Maringá (UNIFAMMA), Brasil. Vale do São Francisco (UNIVASF), Brasil.

RESUMO: O presente estudo objetivou revisar de forma sistemática a literatura atual para sumarizar o efeito de intervenções com dupla tarefa sobre sintomas da Doença de Alzheimer. Trata-se de uma revisão sistemática da literatura. Foram utilizadas as bases de dados PubMed, Scielo e Lilacs. Incluíram-se estudos em idosos com Doença de Alzheimer, em tratamento por meio da dupla tarefa. Os quatro estudos incluídos mostraram que as intervenções duraram de 04 a 12 meses, com frequência semanal de duas a três sessões de no mínimo 60 minutos. O tamanho da amostra variou de 10 a 210 idosos. Um dos estudos apresentou a dupla tarefa com enfoque no exercício funcional. Outros dois apresentaram a dupla tarefa junto de exercícios aeróbios, força, resistência, equilíbrio e coordenação motora. Por fim, o quarto grupo utilizou apenas os exercícios de dupla tarefa. Os estudos têm comprovado que o exercício de dupla tarefa pode amenizar e controlar as perdas motoras e cognitivas ocasionadas por esta doença.

PALAVRAS-CHAVE: Atividade motora; Cognição; Terapia por exercício.

\section{EFFECTS OF DUAL-TASK EXERCISES IN ELDERLY WITH ALZHEIMER'S DISEASE: A SYSTEMIC REVIEW}

\begin{abstract}
A systemic review of current literature is undertaken to summarize the effects of dual-task intervention on the symptoms of Alzheimer's Disease through databases PubMed, Scielo and Lilacs. Studies with elderly with Alzheiomer's Disease treated by the dual-task exercises were also used. The four studies showed that interventions lasted between 4 and 12 months, with weekly frequency of two or three 60-min sessions. Sample greatness varied between 10 and 210 elderly people. One of the studies showed the dual task focused on functional exercise and the other two demonstrated the dual task exercise with aerobic, strength, resistance, equilibrium and motor coordination exercises. The fourth group used the dual task exercise alone. Studies have proved that the dual-task exercise may lessen and control motor and cognitive losses caused by the disease.
\end{abstract}

KEY WORDS: Motor activity; Cognition; Therapy by exercise.

\section{INTRODUÇÃO}

O envelhecimento da população mundial é uma realidade que

Autor correspondente

Daniel Vicentini

d.vicentini@hotmail.com também pode ser observada no Brasil. Presume-se que em 2020 haverá aproximadamente 30,9 milhões de pessoas com 60 anos ou mais, o que significa $14 \%$ de toda a população brasileira, porcentagem essa 
que leva o país ao sexto lugar no ranking mundial ${ }^{1}$.

Com o envelhecimento ocorrem alterações físicas, funcionais, psicológicas, sociais e outras no organismo. O comprometimento cognitivo está entre as maiores preocupações de saúde pública do envelhecimento, podendo evoluir para demência e também se manifestar na deficiência funcional (limitações para atividades básicas de vida diária), associada à perda da independência ${ }^{2}$. Dentre as demências, a Doença de Alzheimer (DA) é a mais prevalente, e está associada ao acúmulo de placas amilóides extraneuronais e emaranhados neurofibrilares intraneuronais e principalmente declínio cognitivo ${ }^{3}$. É subdividida em três fases, são elas: leve, moderada e grave $^{4}$.

No início, a DA compromete a formação hipocampal, memória de curto prazo, com posterior expansão para outras áreas, atingindo a orientação, a atenção, linguagem, capacidade para resolver problemas, habilidades motoras para executar as atividades da vida diária (AVDs), inclusive de higiene pessoal, passando, possivelmente, por todos os estágios, o que exige altos gastos financeiros da família e/ou do poder público ${ }^{2}$. Sabe-se que esta doença compromete também o controle postural, a marcha, a manipulação de objetos, entre outras atividades simples de uso diário, principalmente quando realizadas simultaneamente com uma tarefa cognitiva - Dupla Tarefa (DT) ${ }^{5}$.

Além dos tratamentos farmacológicos e não farmacológicos, a importância de programas de intervenção que conciliem tarefas motoras e cognitivas para melhorar o desempenho motor tem recebido atenção especial ${ }^{6}$. Para indivíduos saudáveis, executar uma tarefa cognitiva simultaneamente a uma motora parece algo simples e automatizado, no entanto, para pacientes com DA é extremamente complexo, exigindo maior atenção para que o desempenho de uma delas ou de ambas não seja comprometido ${ }^{7}$.

Embora muito se pesquise acerca dos benefícios dos exercícios físicos para o tratamento de doenças, Pitkälä et al. ${ }^{8}$ afirmam que ainda faltam mais estudos que investiguem de forma profunda seus efeitos a longo prazo em idosos com demência e, principalmente, se é possível retardar a progressão da incapacidade nesses pacientes e quais os custos desses serviços para a saúde pública, uma vez que pequenos estudos já comprovam estes fatos. Desta forma o presente estudo teve como objetivo revisar de forma sistemática a literatura atual para sumarizar o efeito de intervenções com DT sobre sintomas da DA.

\section{MÉTODOS}

Para a revisão sistemática da literatura foram utilizadas as seguintes bases de dados: PubMed, Scielo e Lilacs. Os descritores para a pesquisa foram: (Alzheimer disease OU alzheimer) E (Exercise OU Physical activity OU Dual task OU cognitive intervention). A busca teve início em março de 2017 e a triagem dos artigos fundamentou-se nos estudos relacionados aos objetivos e aos critérios de inclusão e exclusão descritos a seguir.

Critérios de inclusão: 1) estudos longitudinais - caso-controle (grupo experimental e grupo controle), abertos (grupo experimental) e de coorte; 2) estudos randomizados e não-randomizados; 3 ) amostras constituídas por indivíduos acima de 60 anos e com diagnóstico clínico de DA; e 4) estudos contendo intervenção com exercício físico e atividade cognitiva simultaneamente (DT).

Critérios de exclusão: 1) estudos que não envolviam intervenção por meio de DT; e 2) aqueles em que a amostra não era exclusivamente composta por idosos com DA mas incluía idosos com outras demências ou declínio cognitivo leve.

\section{RESULTADOS}

Após pesquisa com as combinações de descritores mencionados anteriormente, foram encontrados 382 artigos, sendo 107 na base Pubmed, 47 no Scielo e 138 no Lilacs. Destes, 24 foram selecionados de acordo com o título, porém seis foram removidos por duplicata. Após a leitura dos títulos, três artigos foram excluídos por não estarem disponíveis gratuitamente na íntegra. Assim, na leitura dos textos completos, outros 11 foram excluídos por não atenderem aos critérios de inclusão, ou seja, os estudos tratavam de populações com outras demências ou intervenção por meio de outros exercícios. Desta forma, quatro estudos foram incluídos nesta revisão, como mostra a Figura 1. 


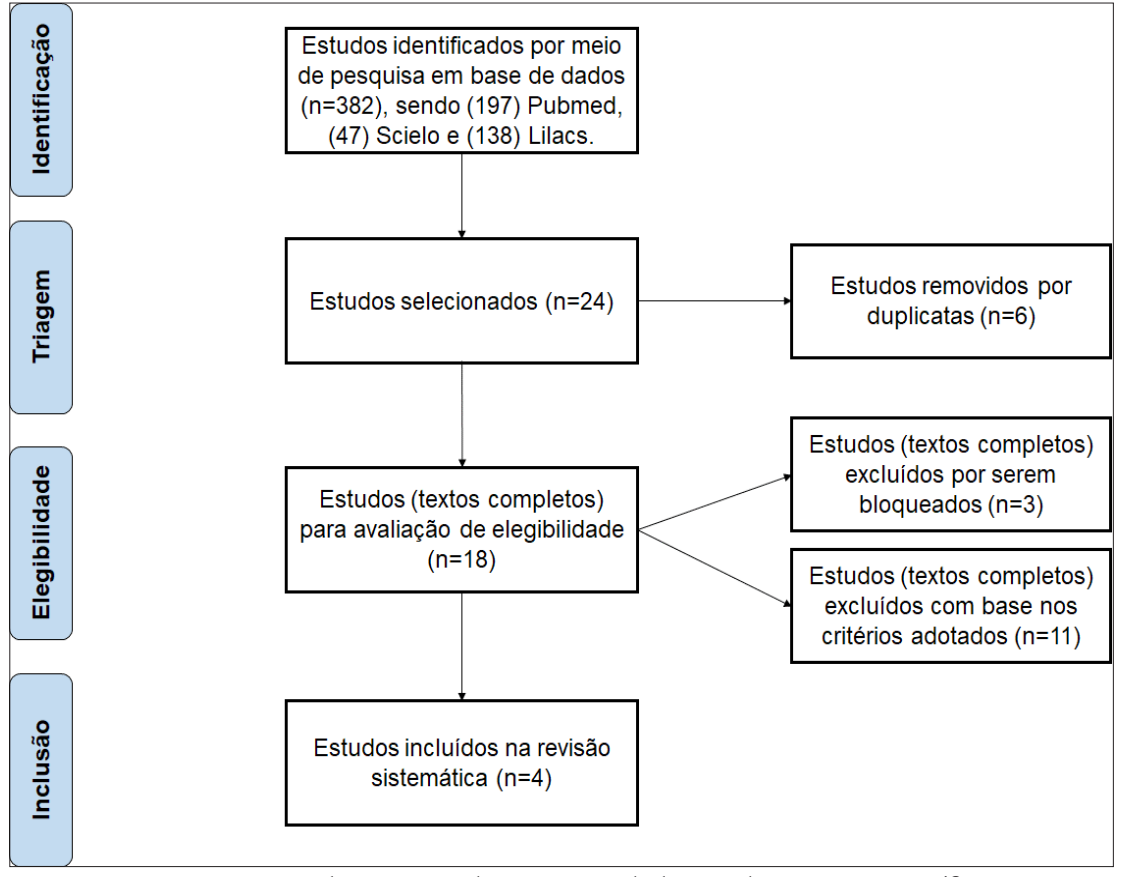

Figura 1. Fluxograma do processo de busca dos artigos científicos

As intervenções duraram de 4 a 12 meses, com frequência semanal de 2 a 3 sessões de no mínimo 60 minutos. O tamanho da amostra variou de 10 a 210 idosos. Quanto à intervenção, um dos estudos apresentou o exercício de DT com enfoque no exercício funcional. Outros dois apresentaram o exercício de DT junto de exercícios aeróbios, força, resistência, equilíbrio e coordenação motora. Por fim, o quarto estudo utilizou apenas os exercícios de DT, como se pode observar no Quadro 1.

Quadro 1. Resumo dos estudos inclusos na revisão sistemática

\begin{tabular}{|c|c|c|c|c|}
\hline Autor, ano & Amostra & Objetivo & Intervenção & Principais Resultados \\
\hline $\begin{array}{l}\text { Fazersztajn; Cordei- } \\
\text { ro; Andreoni; Garcia } \\
(2008)^{9} \text {. }\end{array}$ & $\begin{array}{l}10 \text { idosos } \\
(\text { Grupos com } \\
\text { e sem inter- } \\
\text { venção). }\end{array}$ & $\begin{array}{l}\text { Verificar os efeitos da ativi- } \\
\text { dade física funcional em um } \\
\text { grupo sobre a manutenção } \\
\text { da função motora do pacien- } \\
\text { te idoso com DA como forma } \\
\text { de reabilitação preventiva. }\end{array}$ & $\begin{array}{l}\text { GI (atividades motoras com } \\
\text { enfoque funcional durante } \\
12 \text { semanas) e grupo sem } \\
\text { intervenção. }\end{array}$ & $\begin{array}{l}\text { Houve diferença estatisticamente significativa entre } \\
\text { os dois grupos quanto à mudança média no equilí- } \\
\text { brio. Um aumento significativo de } 1,60 \text { pontos foi } \\
\text { observado no grupo com intervenção nessa escala, } \\
\text { enquanto que no grupo sem intervenção foi de } \\
-0,40 \text {. }\end{array}$ \\
\hline $\begin{array}{l}\text { Nascimento; Teixei- } \\
\text { ra; Gobbi; Gobbi; } \\
\text { Stella }(2012)^{10}\end{array}$ & $\begin{array}{l}20 \text { mulheres } \\
\text { idosas (grupo } \\
\text { com e sem in- } \\
\text { tervenção). }\end{array}$ & $\begin{array}{l}\text { Analisar a influência de um } \\
\text { programa de exercícios de } \\
\text { seis meses sobre transtornos } \\
\text { neuropsiquiátricos e sobre } \\
\text { a performance instrumental } \\
\text { de atividades em pacientes } \\
\text { idosos com DA. }\end{array}$ & $\begin{array}{l}\text { Exercícios aeróbios, exercí- } \\
\text { cios simultâneos envolvendo } \\
\text { resistência, força, flexibilida- } \\
\text { de, coordenação motora e } \\
\text { equilíbrio. } 3 \text { vezes por sema- } \\
\text { na, } 60 \text { minutos por sessão. }\end{array}$ & $\begin{array}{l}\text { O GC apresentou deterioração funcional e neurop- } \\
\text { siquiátrica nas comparações entre tempos pré e } \\
\text { pós-intervenção e entre grupos. }\end{array}$ \\
\hline $\begin{array}{l}\text { Fazersztajn; Cordei- } \\
\text { ro; Andreoni; Garcia } \\
(2008)^{9} \text {. }\end{array}$ & $\begin{array}{l}10 \text { idosos } \\
\text { (Grupos com } \\
\text { e sem inter- } \\
\text { venção). }\end{array}$ & $\begin{array}{l}\text { Verificar os efeitos da ativi- } \\
\text { dade física funcional em um } \\
\text { grupo sobre a manutenção } \\
\text { da função motora do pacien- } \\
\text { te idoso com DA como forma } \\
\text { de reabilitação preventiva. }\end{array}$ & $\begin{array}{l}\text { GI (atividades motoras com } \\
\text { enfoque funcional durante } \\
12 \text { semanas) e grupo sem } \\
\text { intervenção. }\end{array}$ & $\begin{array}{l}\text { Houve diferença estatisticamente significativa entre } \\
\text { os dois grupos quanto à mudança média no equili- } \\
\text { brio. Um aumento significativo de } 1,60 \text { pontos foi } \\
\text { observado no grupo com intervenção nessa escala, } \\
\text { enquanto que no grupo sem intervenção foi de } \\
-0,40 \text {. }\end{array}$ \\
\hline $\begin{array}{l}\text { Fazersztajn; Cordei- } \\
\text { ro; Andreoni; Garcia } \\
(2008)^{9} \text {. }\end{array}$ & $\begin{array}{l}10 \text { idosos } \\
\text { (Grupos com } \\
\text { e sem inter- } \\
\text { venção). }\end{array}$ & $\begin{array}{l}\text { Verificar os efeitos da ativi- } \\
\text { dade física funcional em um } \\
\text { grupo sobre a manutenção } \\
\text { da função motora do pacien- } \\
\text { te idoso com DA como forma } \\
\text { de reabilitação preventiva. }\end{array}$ & $\begin{array}{l}\text { GI (atividades motoras com } \\
\text { enfoque funcional durante } \\
12 \text { semanas) e grupo sem } \\
\text { intervenção. }\end{array}$ & $\begin{array}{l}\text { Houve diferença estatisticamente significativa entre } \\
\text { os dois grupos quanto à mudança média no equilí- } \\
\text { brio. Um aumento significativo de } 1,60 \text { pontos foi } \\
\text { observado no grupo com intervenção nessa escala, } \\
\text { enquanto que no grupo sem intervenção foi de } \\
-0,40 \text {. }\end{array}$ \\
\hline
\end{tabular}

$\mathrm{DA}=$ Doença de Alzheimer; $\mathrm{GC}=$ Grupo Casa; $\mathrm{CD}=$ Centro dia; $\mathrm{MEEM}=$ Mini Exame do Estado Mental; GC = Grupo Controle; GI = Grupo Intervenção. 
No estudo de Fajersztajn et al. ${ }^{9}$ não foram encontradas diferenças significativas entre os grupos nas escalas do Mini Exame do Estado Mental (MEEM), no Inventário Neuropsiquiátrico (NPI-Q), nas AVDs e AIVDs. No entanto, puderam perceber diferenças entre os grupos para equilíbrio. Notou-se ainda uma tendência para que os escores do MEEM fossem mantidos no grupo intervenção, enquanto houve uma queda para o grupo sem intervenção.

Ohman et al. ${ }^{6}$, após analisarem 210 idosos divididos em dois grupos, sendo um grupo com atividades individuais em suas casas (CG) e outro grupo em atividades coletivas em CD, observaram melhora na cognição e mudança no funcionamento executivo para os dois grupos. Porém, não foram encontradas mudanças significativas entre os dois grupos no período de 12 meses em comparação a idade e sexo. Houve uma deterioração durante a intervenção na fluência verbal de todos eles e em contrapartida ocorreu uma diminuição dos escores do MEEM para os dois grupos.

Nascimento et al. ${ }^{10}$ não encontraram diferenças significativas se comparado a idade, sexo e escolaridade nas funções cognitivas no MEEM. No entanto, encontraram correlação moderada entre os distúrbios neuropsiquiátricos e as funções cognitivas. Foi notado que quanto maior o comprometimento cognitivo, menor os escores no MEEM, maiores são os transtornos neuropsiquiátricos. Já em relação aos distúrbios neuropsiquiátricos pré e pós intervenção, o grupo experimental (GE) apresentou melhora enquanto o GC teve uma piora significativa. Nas pontuações totais, pode-se perceber deterioração no GC e estabilidade no grupo experimental.

Na intervenção de Pedroso et al..$^{11}$ foram identificadas diferenças significativas para as funções cognitivas globais confirmando os efeitos do treinamento. O grupo treino apresentou melhor resultado no MEEM após a intervenção. Houve diferença também nas funções executivas, que melhorou no grupo treino, além da melhora no equilíbrio.

\section{DISCUSSÃO}

Os quatro estudos que compuseram esta pesquisa encontraram resultados similares de um modo geral. No MEEM, após a intervenção, foi percebida estabilidade nos grupos que participaram do treino enquanto os grupos que não realizaram atividades apresentaram deterioração cognitiva ${ }^{9 \cdot 11}$. Os resultados obtidos corroboram com o estudo de Andrade ${ }^{5}$, que após intervenção de DT em 14 idosos, encontrou melhores resultados no GI que no GC.

Já o estudo de Ohman et al. ${ }^{6}$ não encontrou diferenças significativas entre os grupos que fizeram diferentes tipos de exercícios. Foram identificadas melhoras nas funções executivas e cognitivas para ambos. Esse achado vai de encontro com o observado em uma revisão narrativa feita por Antunes et al. ${ }^{12}$, que indicou que há melhora nas funções cognitivas em idosos por meio de exercícios aeróbios ou anaeróbios, atividades executivas ou cognitivas, ou seja, os autores defendem que os benefícios são parecidos, independente da modalidade.

Ainda no estudo de Ohman et al. ${ }^{6}$ houve uma deterioração na fluência verbal dos indivíduos dos dois grupos. Este fato pode ter ocorrido por se tratar de um estudo de longo período (12 meses), e, segundo Zanini ${ }^{13}$, a redução da fluência verbal ser uma das características da DA, tendo início insidioso e degradação progressiva.

No estudo de Pedroso et al. ${ }^{11}$ foram observadas diferenças cognitivas significantes entre os grupos, confirmando os benefícios do exercício de DT. Ficou comprovado em dois dos estudos inclusos nesta revisão, que o exercício de DT promove uma melhora também no equilíbrio. Christofoletti ${ }^{14}$ afirma que as atividades cognitivas têm forte influência na manutenção do equilíbrio.

De acordo com o tipo de atividades desempenhadas, a DT pode ser motora, cognitiva ou cognitivo-motora. Nesse sentido, o treinamento de DT é uma estratégia de reabilitação cada vez mais utilizada na prática clínica, que visa facilitar, por meio da realização de atividades funcionais simultâneas, a alocação de recursos atencionais, diminuindo assim a interferência na $\mathrm{DT}^{15-16}$.

Atualmente, os fisioterapeutas têm voltado sua atenção não apenas para o desempenho motor dos pacientes neurológicos, mas também para aspectos cognitivos e relacionados ao contexto ambiental desses indivíduos. As funções executivas, especialmente a atenção 
dividida, verificada pela realização de tarefas concomitantes, têm sido alvo de interesse desses profissionais tanto em estudos observacionais quanto em estudos de intervenção. Isso indica que o desempenho em DT pode ser um item importante tanto na abordagem avaliativa quanto na intervenção fisioterapêutica ${ }^{15-16}$.

Déficits cognitivos e de marcha são sintomas comuns e pioram com a progressão da doença. DT cognitivo-motoras pode ser também um método para investigar a interação entre marcha e cognição e requerem controle motor e cognição para realizar as tarefas. Sabe-se que as tarefas afetam a marcha mesmo em indivíduos saudáveis, reduzindo a velocidade da marcha. A interferência entre o controle motor e a demanda cognitiva é importante para pacientes com deficiência motora em atividades da vida diária. Isso indica que a DT pode ser responsável pelo risco de tropeços e quedas em pacientes com deficiências motoras, devido a outros desafios no controle motor ${ }^{17-19}$.

No presente estudo, uma das ideias é abordar ao leitor a importância da utilização da DT como estratégia de intervenção para promoção da saúde. Apesar do crescimento substancial no número de publicações sobre o tema, poucos estudos tratam especificamente da utilização da DT como recurso terapêutico e ainda há pouca sistematização quanto a esta utilização. Sabendo-se que é um recurso de baixo custo e extremamente rico de possibilidades de aplicação, esse estudo justifica-se em fomentar discussões sobre essa questão.

\section{CONCLUSÃO}

Os estudos têm comprovado que o exercício de DT pode amenizar e controlar as perdas motoras e cognitivas ocasionadas por esta doença. Ainda que bem documentados, os estudos incluídos nesta revisão sugerem que são necessárias mais pesquisas acerca do efeito da prática de DT em idosos com DA, principalmente no que diz respeito ao protocolo utilizado.

\section{REFERÊNCIAS}

1. Veras RP, Oliveira M. Envelhecer no Brasil: a construção de um modelo de cuidado. Ciência \& Saúde Coletiva. 2018; 23 (6): 1929-36.

2. Santos P, Leide C, Ozela PF, Brito MF, Pinheiro AA, Padilha EC, et al. Alzheimer's disease: A review from the pathophysiology to diagnosis, new perspectives for pharmacological treatment. Current medicinal chemistry, 2018; 25 (26): 3141-59.

3. Huang CY, Chen YA, Hwang S Wu RM. Improving Dual-Task Control With a Posture-Second Strategy in Early-Stage Parkinson Disease. Archives of physical medicine and rehabilitation. Arch Phys Med Rehabil. 2018; 99 (8): 1540-46.

4. Whitfield JA, Goberman AM. Speech motor sequence learning: Effect of Parkinson disease and normal aging on dual-task performance. Journal of Speech, Language, and Hearing Research, 2017; 60 (6): 1752-65.

5. Vergara-Diaz G, Osypiuk K, Hausdorff JM, Bonato P, Gow BJ, Miranda JG, et al. Tai Chi for Reducing Dual-task Gait Variability, a Potential Mediator of Fall Risk in Parkinson's Disease: A Pilot Randomized Controlled Trial. Glob Adv Health Med. 2018; 17 (7): $1-12$.

6. Öhman H, Savikko N, Strandberg TE, Kautiainen H, Raivio MM, Laakkonen M, et al. Effects of exercise on cognition: The Finnish Alzheimer disease exercise trial: a randomized, controlled trial. J Amer Geriatr Soc. 2016; 64 (4): 731-8.

7. Gaßner H, Marxreiter F, Steib S, Kohl Z, Schlachetzki J, Adler W, et al. Gait and cognition in Parkinson's Disease: cognitive impairment is inadequately reflected by gait Performance during Dual Task. Front Neurol. 2017; 26 (8): 1-11.

8. Pitkälä KH, Pöyst MM, Laakkonen ML, Tilvis RS, Savikko N, Kautiainen H, et al. Effects of the Finnish Alzheimer disease exercise trial (FINALEX): a randomized controlled trial. JAMA. 2013; 173 (10): 894901.

9. Fajersztajn L, Cordeiro RC, Andreoni S, Garcia JT. Effects of functional physical activity on the mainte- 
nance of motor function in Alzheimer's disease. Dem Neuropsychol. 2008; 2 (3): 233-40.

10. Nascimento CMC, Teixeira CVL, Gobbi LTB, Gobbi S, Stella F. A controlled clinical trial on the effects of exercise on neuropsychiatric disorders and instrumental activities in women with Alzheimer's disease. Braz J Phys Ther. 2012; 16 (3): 197-204.

11. Pedroso RV, Coelho FG, Santos-Galduróz RF, Costa JL, Gobbi S, Stella F. Balance, executive functions and falls in elderly with Alzheimer's disease (AD): a longitudinal study. Arch Gerontol Geriatr. 2012; 54 (2): 348-51.

12. Antunes HK, Santos RF, Cassilhas R, Santos RVT, Bueno OFA, Mello MT. Exercício físico e função cognitiva: uma revisão. Rev Bras Med Esp. 2006; 12 (2): 108-14.

13. Zanini RS. Demência no idoso: aspectos neuropsicológicos. Rev Neuroc. 2010; 18 (2): 220-26.

14. Christofoletti G. Influência da dupla-tarefa no equilíbrio de pacientes com doença de Parkinson e demência do tipo Alzheimer [Tese de Doutorado]. São Paulo: Universidade Estadual de Campinas; 2010.

15. Melzer I, Tzedek I, Or M, Shvarth G, Nizri O, BenShitrit $\mathrm{K}$, et al. Speed of voluntary stepping in chronic stroke survivors under single- and dual-task conditions: a case-control study. Arch Phys Med Rehabil. 2009; 90 (6): 927-33.

16. Mendel T, Barbosa WO, Sasaki AC. Dupla tarefa como estratégia terapêutica em fisioterapia neurofuncional: uma revisão da literatura. Acta fisiátrica, 2015; 22 (4): 206-11.

17. Fritz NE, Cheek FM, Nichols-Larsen DS. Motor-cognitive dual-task training in persons with neurologic disorders: a systematic review. J Neurol Phys Ther (2015) 39 (3): 142-53.

18. Alcock L, Galna B, Lord S, Rochester L. Characterisation of foot clearance during gait in people with early Parkinson's disease: deficits associated with a dual task. J Biomech. 2016; 49 (13): 2763-69.

19. Amboni M, Barone P, Hausdorff JM. Cognitive contributions to gait and falls: evidence and implications.
Mov Disord (2013) 28 (11): 1520-33.

Recebido em: 03/08/2018

Aceito em: 28/12/2018 